\title{
Morpho-Kinematic Analysis of PNe with Intense [N II] and [S II] Emission Lines
}

\author{
H. Riesgo, J. A. López and M. G. Richer \\ Instituto de Astronomía \\ Universidad Nacional Autónoma de México. Ensenada, B. C. México \\ email: hriesgo, jal, richer @astrosen.unam.mx
}

\begin{abstract}
From the sample of 613 PNe used in the paper 'Revised Diagnostic Diagrams for Planetary Nebulae' (Riesgo \& López, 2006) we have isolated a subsample of $51 \mathrm{PNe}$ defined by $\log \left[\mathrm{H} \alpha /\left[\mathrm{N}_{\mathrm{II}}\right]\right]<0$ and $\log \left[\mathrm{H} \alpha /\left[\mathrm{S} \mathrm{II}_{\mathrm{II}}\right]<0.4\right.$. These objects have extremely intense $[\mathrm{N}$ II $]$ and $[\mathrm{S} \mathrm{II}]$ lines with respect to $\mathrm{H} \alpha$ and are indicative of either very low excitation and/or high optical depth conditions, abundance effects in the case of $\mathrm{N}$ or cooling effects in shock excited regions, such as those expected in high velocity, collimated outflows. We have undertaken an imaging and detailed kinematic study of all the members of the subsample visible from the northern hemisphere. In this work we discuss the results and some correlations found between the anomalous line ratios in these objects and their morphological and kinematic characteristics.
\end{abstract}

Keywords. ISM: planetary nebulae

\section{Introduction}

Most of the images and long-slit, high resolution spectra were obtained with the $2.12 \mathrm{~m}$ telescope at the Mexican National Observatory in San Pedro Mártir with the Manchester Echelle Spectrometer (MES-SPM) Meaburn et al. (2003). An exception was SuWt 2, observed at the AAT with the UCLES spectrometer.

The whole sample used by Riesgo \& López (2006) for the line ratios of interest is shown in the left panel of Figure 1. The subsample of 51 PNe defined by $\log [\mathrm{H} \alpha /[\mathrm{N} \mathrm{II}]]<0$ and $\log [\mathrm{H} \alpha /[\mathrm{S} \mathrm{II}]]<0.4$ is shown in the right panel of Figure 1 , where they appear in the lower left corner of the diagram that partially overlaps the supernovae remnant region.

\section{Discussion}

The diagnostic diagrams are a useful tool to identify particular conditions from peculiar line ratios. For example, in this sample of intense [N II] and [S II] lines with respect to $\mathrm{H} \alpha$ we found $\mathrm{H} 2-12$, an object with shock spectra that has been misclassified as a PN and is really a bright, knotty structure in Kepler's SNR (see Riesgo \& López, 2005). The sample also contains KjPn 8 (López et al. 2000) a peculiar PN with high velocity bipolar jets and Sa 2-237, a point-symmetric nebula also with high velocity bipolar outflows (López et al. 2003).

However, and somewhat surprisingly, the sample is not fully dominated by bipolar nebulae or apparent high-speed outflows. Considering our observed sample of $30 \mathrm{PNe}$, plus 6 more objects from the literature, we found that only 19 objects are bipolars, 9 are elliptical, 2 irregular, 2 spherical shells and 1 annular. Two objects have been discarded for not fulfilling the line ratio criteria and one more for not being a true $\mathrm{PN}$.

We find clear evidence of high-speed outflows $\left(>40 \mathrm{~km} \mathrm{~s}^{-1}\right)$ in only 11 objects. $12 \mathrm{PNe}$ are type I (only 5 objects overlap with the previous group). Two $\mathrm{PNe}$ in the 

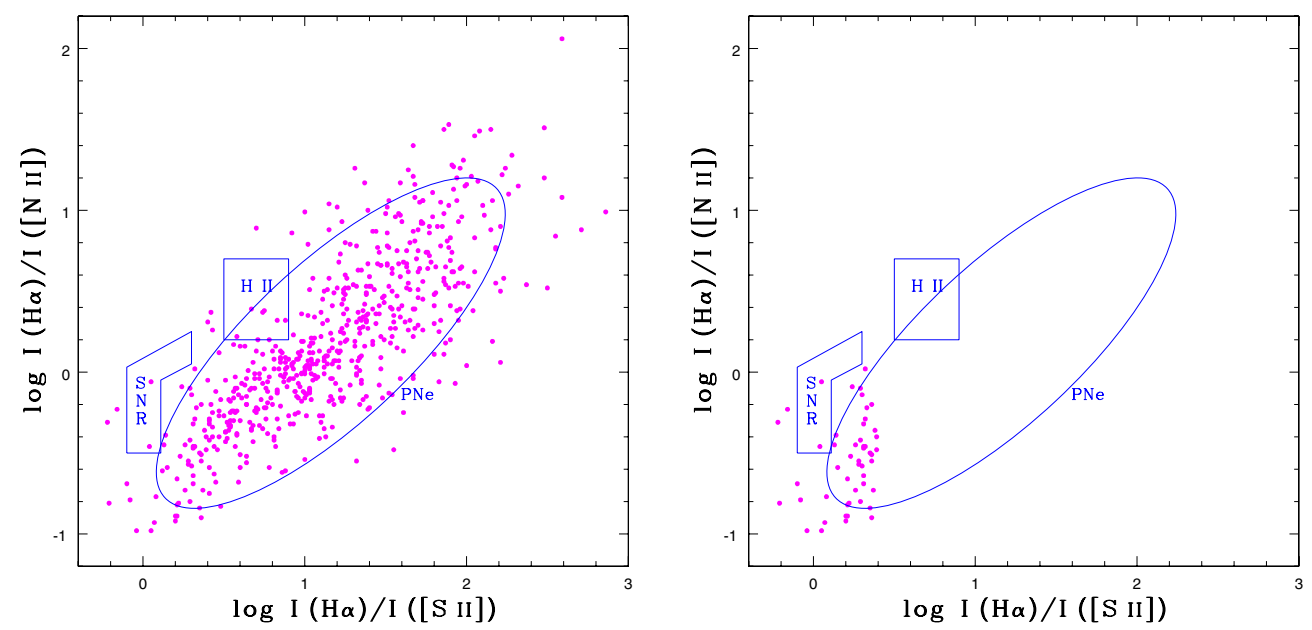

Figure 1. Left: The whole sample used by Riesgo and López (2006). The revised zone for PNe is shown by the ellipse and the boxes show the original limits for H II Regions and SNRs as proposed by Sabbadin et al. (1977). Right: The subsample of 51 PNe defined by $\log [\mathrm{H} \alpha /[\mathrm{N} \mathrm{II}]]<0$ and $\log [\mathrm{H} \alpha /[\mathrm{S} \mathrm{II}]]<0.4$

sample belong to the bulge population. In 8 PNe the [He II] emission line is present in the spectra, indicative of high excitation conditions near the PN core. We did not find additional information for the remaining 15 objects.

\section{Conclusions}

The study shows that the remarkable line ratios that characterize this peculiar sample of $\mathrm{PNe}$ are due to a variety of processes. Collisional cooling, low excitation and high optical depth effects seem to be the major contributors to the high $\mathrm{N}$ and $\mathrm{S}$ lines intensities relative to $\mathrm{H}$. Additional detailed studies on the physical conditions of these objects shall prove of interest in the realm of dynamics, radiation transfer and their link to central star evolution. An extended, journal version of this work will be published shortly.

\section{Acknowledgements}

HR is grateful to CONACYT for a graduate scholarship and to Posgrado-UNAM for a travel grant. We gratefully acknowledge financial support from UNAM-DGAPA grants IN 108406-2, IN 108506 and IN 112103 and CONACYT grant 43121. We also wish to thank the SPM staff for their excellent support.

\section{References}

López, J.A., Meaburn, J., Rodríguez, L.F., Vázquez, R., Steffen, W., \& Bryce, M. 2000, ApJ 538,233

López, J.A., Vázquez, R., Miranda, L.F., Masegosa, J., Meaburn, J., Torrelles, J.M., GarcíaSegira, G. \& Toledano, O. 2003, ASP-CS 303, 441

Meaburn, J., López, J.A., Gutiérrez, L., Quiróz, F., Valdéz, J \& Pedrayez, M. 2005, Rev. Mexicana AyA 39, 185

Riesgo, H. \& López, J.A. 2005, Rev. Mexicana AyA 41, 57

Riesgo, H. \& López, J.A. 2006, Rev. Mexicana AyA 42, 47

Sabbadin,F., Minello, S. \& Bianchini, A. 1977, A\&A 60, 147 